\title{
Author's Accepted Manuscript \\ REACTIVE GAMMA-KETOALDEHYDES AS NOVEL ACTIVATORS OF HEPATIC STELLATE CELLS IN VITRO
}

Lisa Longato, Fausto Andreola, Sean S. Davies, Jackson L. Roberts, Giuseppe Fusai, Massimo Pinzani, Kevin Moore, Krista Rombouts

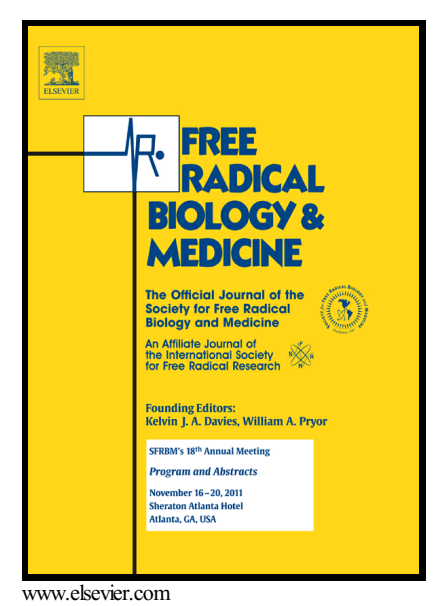

PII: $\quad$ S0891-5849(16)31069-3

DOI: $\quad$ http://dx.doi.org/10.1016/j.freeradbiomed.2016.11.036

Reference: FRB13091

To appear in: Free Radical Biology and Medicine

Cite this article as: Lisa Longato, Fausto Andreola, Sean S. Davies, Jackson L Roberts, Giuseppe Fusai, Massimo Pinzani, Kevin Moore and Krista Rombouts REACTIVE GAMMA-KETOALDEHYDES AS NOVEL ACTIVATORS OF HEPATIC STELLATE CELLS IN VITRO, Free Radical Biology an Medicine, http://dx.doi.org/10.1016/j.freeradbiomed.2016.11.036

This is a PDF file of an unedited manuscript that has been accepted fo publication. As a service to our customers we are providing this early version o the manuscript. The manuscript will undergo copyediting, typesetting, an review of the resulting galley proof before it is published in its final citable form Please note that during the production process errors may be discovered whic could affect the content, and all legal disclaimers that apply to the journal pertain 
Longato

\section{REACTIVE GAMMA-KETOALDEHYDES AS NOVEL ACTIVATORS OF HEPATIC STELLATE CELLS IN VITRO}

Lisa Longato ${ }^{1}$, Fausto Andreola ${ }^{2}$, Sean S. Davies ${ }^{3}$, Jackson L. Roberts ${ }^{3}$, Giuseppe Fusai $^{4}$, Massimo Pinzani ${ }^{1}$, Kevin Moore ${ }^{1^{*}}$, Krista Rombouts $^{1^{*}}$.

${ }^{1}$ Regenerative Medicine \& Fibrosis Group, Institute for Liver \& Digestive Health, University College London, Royal Free, London, UK

${ }^{2}$ Liver Failure Group, Institute for Liver \& Digestive Health, University College of London, Royal Free, London, UK

${ }^{3}$ Department of Pharmacology, Vanderbilt University, Nashville, TN, USA

${ }^{4}$ Division of Surgery, University College London, Royal Free, London, UK

* these authors share joint senior authorship

Corresponding author: Dr. Krista Rombouts. Address: Royal Free Hospital \& University College London UCL, Institute for Liver \& Digestive Health, Division of Medicine, Rowland Hill Street, London NW3 2PF, UK. Tel.: +44 02074332794; fax: +44 02074332852; e-mail: k.rombouts@ucl.ac.uk

Running head: Isolevuglandins activate hepatic stellate cells.

\section{ABSTRACT}

Aims. Products of lipid oxidation, such as 4-hydroxynonenal (4-HNE), are key activators of hepatic stellate cells (HSC) to a pro-fibrogenic phenotype. Isolevuglandins (IsoLG) are a family of acyclic $\gamma$-ketoaldehydes formed through oxidation of arachidonic acid or as by-products of the cyclooxygenase pathway. IsoLGs are highly reactive aldehydes which are efficient at 
Longato

forming protein adducts and cross-links at concentrations 100-fold lower than 4hydroxynonenal. Since the contribution of IsoLGs to liver injury has not been studied, we synthesized $15-E_{2}$-IsoLG and used it to investigate whether IsoLG could induce activation of HSC. Results. Primary human HSC were exposed to $15-E_{2}-$ IsoLG for up to 48 hours. Exposure to $5 \mu \mathrm{M} 15-\mathrm{E}_{2}$-IsoLG in HSCs promoted cytotoxicity and apoptosis. At non-cytotoxic doses (50 pM-500 nM) 15- $\mathrm{E}_{2}$-IsoLG promoted HSC activation, indicated by increased expression of $\alpha$-SMA, sustained activation of ERK and JNK signaling pathways, and increased mRNA and/or protein expression of cytokines and chemokines, which was blocked by inhibitors of JNK and NF-kB. In addition, IsoLG promoted formation of reactive oxygen species, and induced an early activation of ER stress, followed by autophagy. Inhibition of autophagy partially reduced the pro-inflammatory effects of IsoLG, suggesting that it might serve as a cytoprotective response. Innovation. This study is the first to describe the biological effects of IsoLG in primary HSC, the main drivers of hepatic fibrosis. Conclusions. IsoLGs represent a newly identified class of activators of HSC in vitro, which are biologically active at concentrations as low as $500 \mathrm{pM}$, and are particularly effective at promoting a pro-inflammatory response and autophagy.

\section{KEYWORDS}

Human hepatic stellate cells; fibrosis; isolevuglandins; isoketals; gamma-ketoaldehydes; inflammation; autophagy; endoplasmic reticulum stress; oxidative stress.

\section{INTRODUCTION}

Activation of hepatic stellate cells (HSC) to a myofibroblastic phenotype is a key event in the response of liver tissue to acute and chronic injury. Activated HSC are programmed for the 


\section{Longato}

synthesis, deposition and remodeling of collagens and other extracellular matrix (ECM) components in order to re-constitute the basal ECM necessary for tissue regeneration. However, the chronic activation of this process through persistence of parenchymal injury, causes the progressive accumulation of fibrillar ECM leading to liver cirrhosis [1-3]. An established trigger for HSC activation is oxidative stress, and, in particular, the products of lipid oxidation or oxidative modification of membrane polyunsaturated fatty acids. This class of molecules includes the reactive lipid aldehydes malondialdehyde, 4-hydroxy-2-nonenal (4$\mathrm{HNE}$ ), and $\mathrm{F}_{2}$ isoprostanes (such as 8-iso-PGF2 $\alpha$ ) $[4,5]$. Importantly, exposure of HSC to 4HNE leads to increased synthesis and deposition of collagen I [6, 7], increased expression of matrix remodeling proteins, such as TIMP1 [7], activation of JNK signaling pathway [8], and increased pro-inflammatory signaling (MCP-1) [9]. Altogether these data suggest that products of lipid oxidation have a direct pro-inflammatory and pro-fibrogenic effect on HSC, the main cellular effectors of liver fibrogenesis.

Despite the wealth of data supporting a role for 4-HNE as a key pro-fibrogenic stimulus, and thus potentially important in the pathogenesis of chronic liver disease, the role of other closely related bioactive molecules, such as IsoLGs, has not been addressed. This class of molecules includes 8 regioisomers of acyclic $\gamma$-ketoaldehydes collectively referred to as isolevuglandins (IsoLGs, also called Isoketals or IsoK) [10, 11]. IsoLGs are formed either through free radical-mediated oxidation of arachidonic acid or during the formation of prostaglandins by cyclooxygenase. Non-enzymatic rearrangement of the bicycloendoperoxide intermediates of these two pathways $\left(\mathrm{PGH}_{2}\right.$ or its regioisomers, respectively) yield acyclic $\gamma$ ketoaldehydes. Whilst the free-radical mediated pathway generates a mixture of regio- and stereoisomers, the rearrangement of $\mathrm{PGH}_{2}$ yields a single stereoisomer of $15-\mathrm{E}_{2}-\mathrm{IsoLG}$ and 15 $D_{2}$-IsoLG, designated as $L G E_{2}$ and $L G D_{2}$ respectively $[12,13]$. 


\section{Longato}

Unlike 4-HNE, which mainly reacts with cysteine [14], IsoLGs are selectively reactive towards lysine residues, leading to the formation of stable lactam and hydroxylactam adducts [15]. The rate of adduction for IsoLGs is several folds higher than the one of $4-\mathrm{HNE}$, so that IsoLGs are considered some of the most reactive products of lipid peroxidation [16]. Of note, due to such rapidity of adduction, these compounds are virtually undetectable in free form in biological systems containing proteins [16]. In addition, IsoLGs induce protein crosslinks and oligomerization at concentrations at least 100 -fold lower than those required for 4 -HNE to promote the same degree of crosslinking $[14,17]$. The formation of IsoLGs protein adducts may result in loss of function, formation of neo-antigens, and inhibition of proteasome function [18]. IsoLGs have also been shown to form adducts with nucleic acids [19] and lipids, such as phosphatidyl-ethanolamine, resulting in the induction of endoplasmic reticulum stress, and mitochondrial dysfunction [20, 21]. Other cellular consequences of IsoLGs exposure include increased expression of pro-inflammatory cytokines and adhesion molecules [20], and cell death $[18,22]$.

In vivo, IsoLGs are formed during inflammation, either through increased formation of prostaglandins and re-arrangement of the $\mathrm{PGH}_{2}$ intermediate formed by cyclooxygenase [23], or during oxidative stress [24, 25]. Consistent with this observation, circulating and tissue levels of IsoLGs adducts are increased in multiple disease conditions associated with both, including end-stage renal disease and atherosclerosis [26], hypertension [27], pulmonary fibrosis [22]. For this reason, IsoLGs have been proposed as both markers and mediators of oxidative damage.

With respect to their potential role in liver disease, IsoLGs adducts have been described in livers of rodents exposed acutely to carbon tetrachloride [16], and chronically to ethanol [28, 29], or high fat diet [30]. However, IsoLGs's effects on specific hepatic cell types and their potential contribution to disease pathogenesis have not been investigated. In this work we 
Longato

sought to characterize the biological effects of a synthetic form of the model IsoLGs $15-\mathrm{E}_{2^{-}}$ levuglandin (15- $\mathrm{E}_{2}$-IsoLG) on human HSC.

\section{MATERIALS AND METHODS}

Sources of reagents. $15-E_{2}$-levuglandin $\left(15-E_{2}-\right.$ IsoLG) was synthesized as previously described and supplied in DMSO (10 mM) [31]. In brief, $100 \mu \mathrm{l}$ of a $10 \mathrm{mM}$ solution of the stable precursor 8-Acetyl-9-dimethoxymethyl-12-hydroxyheptadeca-5(Z),10(E)-dienoic acid in dichloromethane was incubated with Montmorillonite K-10 (10 mg) under slight agitation for 15 minutes at room temperature in an Eppendorf tube. After 15 minutes the clay was separated by centrifugation (16000g), and the supernatant collected, the residual clay was rinsed with 2 $x 500 \mu \mathrm{L}$ of dichlormethane, re-centrifuged, and the supernatant added to the initial supernatant. Lastly, $200 \mu \mathrm{L}$ of DMSO was added, and the dichloromethane removed under a stream of nitrogen to yield a 3-4 mM solution of $15-E_{2}$-IsoLG, as measured via spectrophotometry at 580 nm (Omega FLUOstar microplate reader (BMG Labtech, Ortenberg, Germany). Each batch of $15-E_{2}$-IsoLG was prepared in an identical manner, yielded similar biological effects between experiments, and was stored up to 8 weeks at $-80^{\circ} \mathrm{C}$. For each batch, functional read-outs, such as metabolic activity and proliferation were tested in different human hepatic stellate cells preparations and showed no variation. As this reagent is not available commercially, it will be provided upon request (Dr S. Davies, Vanderbilt University, US). For experiments involving chemical inhibitors, hHSC were pre-incubated for one hour with inhibitors of pan JNK (SP600125, $20 \mu \mathrm{M})$, p38 MAPK (SB203580, $10 \mu \mathrm{M})$, and NF-kB (Pyrrolidinedithiocarbamate, PDTC, $50 \mu \mathrm{M}$ ) (TOCRIS Biosciences, Bristol, UK). For autophagy induction/inhibition chloroquine and bafilomycin were used at $15 \mu \mathrm{M}$ and $10 \mathrm{nM}$ concentrations, respectively. Unless otherwise specified, all other reagents were from Sigma (Dorset, England). 


\section{Longato}

Human hepatic stellate cells (hHSC) isolation and culture. Cells were isolated from resected livers wedges, obtained from patients undergoing surgery at the Royal Free Hospital after giving informed consent (EC01.14-RF). Cells were isolated according to a published protocol [32], with modifications for human liver [2]. In brief, 10 grams of total human liver tissue were digested with $0.01 \%$ Collagenase, $0.05 \%$ Pronase, and $0.001 \%$ DNase I without perfusion. The homogenate was filtered through a $100 \mu \mathrm{m}$ cell strainer (BD Falcon,Oxford, UK), and the flow-through was centrifuged at $50 \times \mathrm{xg}$ for 2 minutes at $4^{\circ} \mathrm{C}$. After washing the supernatant, gradient centrifugation was performed at $1400 \mathrm{xg}$ for 17 minutes at $4^{\circ} \mathrm{C}$ using an $11.5 \%$ Optiprep gradient (Sigma). Finally, the interface was collected and washed. Purity of the obtained hHSC was confirmed by detection of CD140b (PDGFRbeta), CD29 (Integrin beta 1) and Cytoglobin B (CYGB). The obtained HSC were cultured in Iscove's Modified DMEM (IMDM), supplemented with $20 \%$ foetal bovine serum (FBS), $2 \mathrm{mM}$ Glutamine, $1 \mathrm{X}$ nonessential amino acids, $1.0 \mathrm{mM}$ sodium pyruvate, 1X antibiotic-antimycotic (all from Life Technologies, Paisley, UK), referred to as complete HSC medium hereinafter. Each HSC preparation was maintained under standard conditions in a humidified incubator under $5 \% \mathrm{CO}_{2}$ in air at $37^{\circ} \mathrm{C}$. Experiments described in this study were performed on hHSC of at least three independent cell preparations, used between passage 3 and $8[8,33]$.

$15-E_{2}$-IsoLG in vitro treatments. For all the described experiments, HSC cultures were cultured in serum-free (SFM) medium for $24 \mathrm{~h}$ before the experimental treatment. The day of the treatment, cells were first exposed to $15-\mathrm{E}_{2}$-IsoLG diluted in HBSS containing $\mathrm{Ca}^{2+} / \mathrm{Mg}^{2+}$ for 15 , followed by incubation of $15-E_{2}$-IsoLG diluted in SFM for the remaining duration of the experiment. The pre-incubation in amino acids-free media (HBSS) was aimed at minimizing the possibility of $15-E_{2}$-IsoLG adduction to culture media components [18, 34]. The maximum duration of the pre-incubation period was established in preliminary experiments, which demonstrated that HBSS was well tolerated, and did not elicit substantial metabolic effects in primary HSC. 


\section{Longato}

The highest concentration of DMSO (15- $\mathrm{E}_{2}$-IsoLG vehicle) in the diluted $15-\mathrm{E}_{2}$-IsoLG (at the $5 \mu \mathrm{M}$ dose) was $\leq 0.05 \%$. For all the other doses, which were prepared by serial dilution, the DMSO concentration was always $\leq 0.01 \%$, the effect of which was considered negligible. Therefore, control samples, all pre-treated with HBSS, have been labeled as SFM only. The volumes/well used for cell treatments were respectively: $3 \mathrm{ml}$ (6-wells plates), $1 \mathrm{ml}$ (24-wells plates), $0.5 \mathrm{ml}$ (multiwell chamber slides), and $200 \mu \mathrm{l}$ (96-wells plates).

Dose-response studies. hHSC were seeded at a density of $6-6.5 \times 10^{4} \mathrm{cells} / \mathrm{ml}$ in $96-$ well plates and treated, in quadruplicates, with $15-\mathrm{E}_{2}$-IsoLG serially diluted, so that in all resulting treatments the concentrations of DMSO were always $\leq 0.05 \%$. The wide range of 15 $\mathrm{E}_{2}$-IsoLG concentrations tested ( $\left.5 \mu \mathrm{M}-5 \mathrm{pM}\right)$, was chosen as it encompasses the concentrations at which 4-HNE or 8-epi-PGF2 $\alpha$ maximally activate HSCs, according to existing literature [4, 6, 7]. 24-48 hours after treatment, viability/cytotoxicity were measured via MTS assay (Promega, Madison, WI), Neutral red assay [35], and lactate dehydrogenase (LDH) quantification in culture supernatants (Thermoscientific, Pierce, Loughborough, UK). In parallel experiments, the effect of $15-E_{2}$-IsoLG on DNA synthesis was measured via colorimetric BrdU ELISA (Roche, UK). For the subsequent experiments, $15-\mathrm{E}_{2}$-IsoLG was used at concentrations of $0.5 \mu \mathrm{M}-5 \mathrm{nM}$.

RNA extraction and qRT-PCR. Total RNA was extracted from 6-wells cultures treated with 15- $E_{2}$-IsoLG for 24 hours via TRIzol reagent, and RNeasy Universal Mini Kit (Qiagen,Manchester, UK). One microgram of total RNA was reverse transcribed with random primers and MultiScribe RT enzyme (Applied Biosystems, Paisley,UK). Taqman ${ }^{\circledR}$ gene expression assays were used to measure via qRT-PCR the levels of the transcripts for markers of fibrogenic activation, selected chemokines/cytokines, and adhesion molecules (Table 1). Signal was acquired with Applied Biosystems 7500 Fast Real-Time PCR System (ThermoFisher Scientific, Paisley, UK), and data were analyzed using the delta delta Ct method [36]. Levels of transcripts of interest were normalized to the ones of GAPDH as reference gene. 


\section{Longato}

HEK-Blue $^{\mathrm{TM}}$ IL-18/IL-1 $\beta$ assays. Production of bioactive IL-1ß/IL-18 was assessed via using HEK-Blue ${ }^{\mathrm{TM}}$ IL-18/IL-1 $\beta$ reporter cells, which carry the secreted embryonic alkaline phosphatase (SEAP) gene as reporter (InVivoGen, Toulouse, France). In brief, reporter cells were incubated for 24-48 hours with conditioned media from HSCs treated with $15-\mathrm{E}_{2}$-IsoLG for 48 hrs. 24-48 hours later, alkaline phosphatase activity was measured via Quanti-Blue reagent (InVivoGen, Toulouse, France). Absorbance at $620-655 \mathrm{~nm}$ was read in the Omega FLUOstar microplate reader (BMG Labtech, Ortenberg, Germany).

Western Blot. Cells lysates from 6-wells cultures treated with $15-E_{2}$-IsoLG for $24-48$ hours were prepared in radioimmunoprecipitation (RIPA) buffer containing $20 \mathrm{mM}$ Tris/ $\mathrm{HCl}, \mathrm{pH}$ 7.4, $150 \mathrm{mM} \mathrm{NaCl}, 5 \mathrm{mM}$ EDTA, 1\% Nonidet P-40, 1 mM Na $\mathrm{VO}_{4}, 1 \mathrm{mM}$ PMSF, 1:100 protease inhibitor cocktail (Sigma). Cell debris were pelleted by centrifugation at $10,000 \mathrm{rpm}$ at $4{ }^{\circ} \mathrm{C}$ for 15 minutes and total proteins were measured via bicinconinic (BCA) assay (Pierce, Rockford, IL,USA) and stored at $-80^{\circ} \mathrm{C}$ for further analysis. $20-25 \mu \mathrm{g}$ of protein extracts were separated by SDS-PAGE with $8-12 \%$ acrylamide gel and transferred to a PVDF membrane (Millipore, Bedford, USA). After blocking for $1 \mathrm{~h}$ with $3 \%$ bovine serum albumin (BSA), membranes were incubated overnight $4{ }^{\circ} \mathrm{C}$ under mild agitation with primary antibody solutions (1\% BSA, TBS $1 \mathrm{X}$ and $0.1 \%$ Tween 20). Membranes were then washed with TBS $1 \mathrm{X} / 0.1 \%$ Tween 20 , followed by incubation with the appropriate secondary HRP-conjugated antibody. Protein immunoreactivity was revealed with the ECL system (Thermoscientific, Pierce), using a FluorChem M (Protein Simple, San Jose, USA). To demonstrate equal loading of protein samples, membranes were stripped with stripping buffer and were re-probed with tubulin antibody. The complete list of antibodies used is provided in Table 2.

\section{Measurement of intracellular reactive oxygen and nitrogen species formation.}

HSC (20000/well) were grown to sub-confluency on multi-well chamber slides (Millipore, Watford, UK), serum starved for 24 hours, then treated with $0.5 \mu \mathrm{M} / 50 \mathrm{nM}$ of $15-\mathrm{E}_{2}$-IsoLG for 


\section{Longato}

one hour. Formation of reactive oxygen species (ROS) and reactive nitrogen species (RNS), as a general indicator of oxidative stress, was measured by incubation with the 5-(and-6)-carboxy2',7'- dichlorodihydrofluorescein diacetate (Carboxy- $\mathrm{H}_{2}$ DCFDA) fluorescent probe (ThermoFisher Scientific, Paisley, UK). Total cell distribution was revealed by staining nuclei with the Hoechst 33342 dye (final concentration of $1 \mu \mathrm{M}$ ). Images were acquired via fluorescence microscope (AxioScopeA1, Carl Zeiss Ltd).

Transmission Electron Microscopy. hHSC were grown on glass coverslips and treated with $15-E_{2}$-IsoLG for $24-48$ hours as previously described. After treatment, cells were fixed in $1.5 \%$ glutaraldehyde, $2 \%$ paraformaldehyde in $0.1 \mathrm{M}$ cacodylate buffer ( $\mathrm{pH} 7.4$ ) for 24 hours at $4^{\circ} \mathrm{C}$. After two washes in cacodylate buffer, the cells were post-fixed in $1 \%$ osmium tetraoxide $/ 1.5 \%$ potassium ferrocyanide in $0.1 \mathrm{M}$ cacodylate buffer at $4^{\circ} \mathrm{C}$ for 45 minutes. After an additional wash in cacodylate buffer, and a rinse in $\mathrm{dH}_{2} \mathrm{O}$, the cover slips were dehydrated in a graded ethanol-water series, and infiltrated with Agar 100 resin for 24 hours. The cover slip was then removed using hydrofluoric acid. A representative area was selected and ultra thin sections were cut at $70-80 \mathrm{~nm}$, using a diamond knife on a Reichert ultracut $\mathrm{S}$ microtome. Sections were collected on 200 mesh copper grids, stained with lead citrate, and viewed with a Joel 1010 transition electron microscope. Images were recorded using a Gatan Orius CCD camera (Gatan, UK).

Immunofluorescence. hHSC (20000/well) grown on multi-well chamber slides (Millipore, Watford, UK) were treated with 15- $E_{2}$-IsoLG for 24 hours. After methanol fixation, rinsing and blocking steps, slides were incubated with monoclonal anti-mouse LC3B antibody, followed by AlexaFluor 488-conjugated secondary antibody (ThermoFisher Scientific, Paisley, UK). Slides were mounted with the DAPI-containing mounting medium Vectashield (Vector, Peterborough, UK) and imaged with fluorescence microscope (AxioScopeA1, Carl Zeiss Ltd). Negative controls included were obtained by omitting the primary or the secondary antibody 
Longato

from the protocol. For PDI immunofluorescence a similar protocol was followed, except for the use of $4 \%$ paraformaldehyde as fixative and of an Alexa Fluor 488 conjugated PDI antibody (Cell Signaling).

Data analyses. All analyses were performed in at least 3 different HSC preparations. Data were analyzed with Prism software (Graph Pad, CA, USA) using either one-way or twoways ordinary ANOVA, with either Dunnett's or Bonferroni's multiple comparisons tests.

\section{RESULTS}

Determination of the range of bioactive concentrations of IsoLG in HSC. The first goal was to determine if IsoLGs promote similar phenotypic effects in terms of cytotoxicity and proliferation on HSC activation to those described for 4-HNE and isoprostanes, and at comparable doses. HSC treated with complete medium (CM) were used as positive controls, since serum supplementation increases proliferation and metabolic activity (Fig 1C-H). After 24 hours, exposure to $5 \mu \mathrm{M} 15-\mathrm{E}_{2}$-IsoLG resulted in profound cytotoxicity, as indicated by increased LDH leakage in cell culture media (Fig.1A), and reduced retention of Neutral Red staining (Fig 1E) (38). Despite the profound cytotoxicity observed, at 24 hours, formazan reduction (MTS), an indicator of mitochondrial function was not significantly reduced (Fig 1C), which could indicate a compensatory response. After 48 hours, the severity of these changes was even higher, with significantly increased LDH leakage in cell culture media (1B), and significantly reduced MTS and Neutral Red staining compared to control cells (1B, D, F). In contrast, at doses from $1 \mu \mathrm{M}-5 \mathrm{nM}$ of $15-\mathrm{E}_{2}$-IsoLG no significant metabolic or toxic effects were observed, at either of the time-points considered $(1 \mathrm{~A}-\mathrm{F})$. Further, since reactive lipid aldehydes have a mixed effect on cellular proliferation, we assessed the effects of $15-E_{2}$-IsoLG exposure on DNA synthesis by measurement of BrdU incorporation. Consistent with the severe toxicity 


\section{Longato}

and cell loss observed in the highest dose of $15-E_{2}$-IsoLG used, BrdU incorporation was significantly reduced at both at 24 and 48 hours $(1 \mathrm{G}, \mathrm{H})$. At all the other doses considered, IsoLG exposure did not increase BrdU incorporation, suggesting a lack of effect on HSC proliferation, both at 24 and $48 \mathrm{hrs}(1 \mathrm{G}, \mathrm{H})$. Collectively, these data suggest that, over the broad range of concentrations tested, $15-\mathrm{E}_{2}$-IsoLG was profoundly cytotoxic at $5 \mu \mathrm{M}$ dose, but, at lower doses, did not promote hHSC proliferation.

High doses of IsoLG promote apoptotic cell death in HSC. To determine the mechanism of high dose IsoLG induced cytotoxicity we exposed hHSC to $5 \mu \mathrm{M} 15-\mathrm{E}_{2}$-IsoLG for up to 24 hours. Morphologically, cytotoxicity was evident already after 4 hours (data not shown). Western blot analyses confirmed that the observed cytotoxicity was due to induction of apoptotic cell death, as demonstrated by time-dependent activation of the classical markers of apoptosis cleaved PARP, as well as the phosphorylation and activation of the pro-apoptotic signaling pathway JNK (Fig. 2). Since IsoLG cytotoxicity has been associated with adducts formation, inhibition of proteasome function and activation of the UPR response, we measured the ER stress dependent pro-apoptotic transcription factor CHOP/GADD153 [18, 20]. Indeed, IsoLG exposure promoted a robust up-regulation of the pro-apoptotic transcription factor GADD153/CHOP, which preceded even the increases of other markers of cell death.

Effects of non-cytotoxic doses of IsoLG on activation of signaling pathways and markers of HSC activation. Despite the apparent lack of metabolic or pro-proliferative effects at the lower doses of $15-E_{2}$-IsoLG studied, we sought to determine the extent of phenotypic changes in HSC, with focus on multiple features of HSC activation. For these experiments, hHSC were treated with a range of non-cytotoxic doses (500 nM- $0.5 \mathrm{nM})$ of 15- $\mathrm{E}_{2}$-IsoLG for 24 hours. As the main fibrogenic effect of reactive lipid aldehydes is via increased collagen 1 mRNA production [6], we sought to assess the effects of IsoLG exposure on key genes in collagen synthesis and maturation, as well as other matrix remodeling enzymes, such as tissue 


\section{Longato}

inhibitor of metalloprotease 1 and lysyl oxidase (Fig. 3). 15- $E_{2}$-IsoLG exposure was not associated with any increase in COL1A1 mRNA levels, whilst, at the highest dose used, it actually led to a significant decrease. Similarly, 15- $E_{2}$-IsoLG exposure had no effect across all concentrations tested in the modulation of TIMP-1 and LOX (Fig.3).

Next we sought to determine the effects of IsoLG on some signaling pathways critical to HSC biology, which are known to be modulated by exposure to reactive lipid aldehydes, such as 4-HNE, namely mitogen activated protein kinases (MAPKs), including p38 MAPK, extracellular signal-regulated kinases 1 and 2 (ERK1/2), and c-Jun NH2-terminal kinase (JNK) [7, 8]. Exposure to IsoLG promoted a robust activation of extracellular related kinase $1 / 2$ (ERK) particularly at the two highest concentrations used. Likewise, we observed a strong upregulation of JNK phosphorylation at the same concentrations. In contrast, at 24 hours we detected no significant activation of p38 MAPK. In addition, we demonstrate that IsoLG exposure caused increased expression of alpha smooth muscle actin, a classical marker of myofibroblastic activation.

Exposure to IsoLG promotes increased cytokines/chemokines production, and intracellular ROS production in HSC. In other cell types, IsoLGs exposure promotes a proinflammatory phenotype, via upregulation of adhesion molecules, chemokines/cytokines, and transcription factors $[20,27,30]$. Therefore, we investigated if incubation of HSCs with IsoLGs had similar effects by measuring mRNA levels of multiple pro-inflammatory mediators [37]. Indeed, IsoLGs treated HSCs exhibited significantly increased levels of the adhesion molecule ICAM-1 at the $50 \mathrm{nM}$ dose. In addition, IsoLG exposure resulted in significantly elevated levels of IL-6, as well as of the chemokine MCP1/CCL2 across all the range of concentrations tested. In the case of IL-8, exposure to $500 \mathrm{nM}$ and $50 \mathrm{nM}$ IsoLG resulted in a 15-fold and 5-fold increase in mRNA levels, respectively, compared to control cells. Likewise, IL-1 $\beta$ levels were approximately 8-fold higher at the highest dose examined. These changes in RNA levels led to a significant increase of secreted and bioactive IL-1 $1 \beta$, as measured via a reporter assay (Figure 


\section{Longato}

4F). To determine which signaling pathways are responsible for such robust increases in proinflammatory cytokine production, in a subset of experiments, HSC were pre-incubated for one hour with chemical inhibitors of JNK, p38 MAPK, and NF-kB, prior to treatment with IsoLGs's for 24 hours as previously described. As before, cells treated with IsoLGs displayed a robust increase in IL-1 $\beta$ levels over vehicle treated cells, which was completely abrogated by treatment with JNK inhibitor, but not by p38 MAPK. Despite a lack of detectable activation of NF-kB signaling via western Blot (data not shown), treatment of cells with the NF-kB inhibitor PDTC also partially prevented the increase in IL-1 $\beta$ levels. Overall, inhibition of JNK was the most effective at reducing overexpression of pro-inflammatory cytokines, whereas p38 MAPK was the least effective (Fig 4). Collectively, these results indicate that in human HSC IsoLGs trigger proinflammatory effects, by stimulating cytokine and chemokine production and secretion, which is largely dependent upon JNK activation.

It has been proposed that activation of JNK kinases following reactive lipid aldehydes exposure (4-HNE) could be mediated by redox changes, in particular from increased ROS formation [38]. Indeed exposure to 4-HNE results in increased ROS production in a number of cell types [38-40]. Further, IsoLGs are known to cause mitochondrial dysfunction in isolated mitochondria, another potential source of ROS [21]. Therefore we sought to determine if $15-\mathrm{E}_{2^{-}}$ IsoLG may also promote increased ROS formation in HSC, using the fluorescent probe DCFDA. For these experiments we focused on the early changes (1 hour), as previously demonstrated [39]. After one hour of incubation with $15-E_{2}$-IsoLG, there was a dose dependent increase of fluorescence at the two doses tested, compared to SFM treated cells, suggesting increased production of intracellular ROS (Figure 4).

Exposure to IsoLG promotes increased autophagy in HSC. Our results so far indicate that IsoLG exposure in HSC induces stress signaling, pro-inflammatory changes, and redox changes. Mechanistically, in other cell types, IsoLG act primarily by forming adducts to 


\section{Longato}

protein, lipids or nucleic acids [41]. Notably, IsoLG-adducted proteins are poor substrates for proteosomal degradation and the proteasome itself can be functionally impaired following IsoLG exposure [18]. We then thought that some of the effects observed in HSC could be mediated by induction of autophagy, an alternative pathway to the ubiquitin-proteasome system to remove damaged proteins and organelles [42, 43]. To determine whether autophagy was present IsoLGs treated cells, we performed immunofluorescent staining for the autophagy marker LC3B, a protein involved in autophagosome formation[44]. While control cells displayed low abundance of LC3B puncta, HSCs treated with the autophagy inducer chloroquine (15 $\mu \mathrm{M})$ as positive control had abundant distribution of LC3B puncta throughout the cytoplasm (Fig 5B). 15- $\mathrm{E}_{2}$-IsoLG treated cells had an intermediate abundance of autophagic vesicles. Ongoing autophagy was further confirmed by LC3B detection via Western Blot analysis demonstrating increased conversion of LC3B in the autophagosome localized, phosphatidylethanolamine conjugated, LC3B II isoform (Fig 5C). As an additional approach to confirm the presence of autophagy we conducted ultrastructural analyses in HSC treated with non-cytotoxic doses of IsoLGs for 24 and 48 hours. Electron microscopy revealed increased presence of autophagosomes containing morphologically intact cytosol or organelles (Figure $5 \mathrm{~A}$ ) in $15-\mathrm{E}_{2^{-}}$ IsoLG treated cells, compared to control cells after 24 hours. After 48 hours, such autophagic structures appeared even more mature, containing partially degraded cytoplasmic, as well as organelle material. We then wondered whether the functional significance of autophagy induction in IsoLG treated cells could be a compensatory response to reduce proteotoxic stress. To test this hypothesis we simultaneously exposed cells to $15-E_{2}$-IsoLG and to the autophagy inhibitor bafilomycin at concentrations previously used in HSC [45]. Treatment of HSC with 10 $\mathrm{nM}$ bafilomycin induced a robust increase of LC3BII levels, consistent with its reduced turnover resulting from a block of autophagosome fusion with lysosome, thus failure to complete autophagy $[45,46]$ In addition, bafilomycin treatment in control cells increased levels of IL1 $\beta$ 


\section{Longato}

mRNA. However, bafilomycin treatment in 15- $\mathrm{E}_{2}$-IsoLG-treated cells profoundly and dosedependently upregulated IL1 $\beta$ mRNA levels, suggesting the beneficial effect of autophagy in curbing proteotoxicity. Collectively, these results demonstrate that IsoLG exposure in HSC results in induction of cellular autophagy.

\section{Exposure to IsoLG promotes early activation of ER stress, which precedes} autophagy in HSC. One of the triggers for autophagy is ER stress, and secondary activation of the unfolded protein response (UPR) [47, 48]. Indeed activation of the unfolded protein response has previously been described in HUVEC cells following exposure to IsoLG [20]. Therefore we sought to determine whether the effects of IsoLG's on autophagy were preceded by an increase in ER stress, and UPR activation. Since ongoing ER stress is associated with ER enlargement and increased synthesis of protein folding machinery, we undertook timecourse analysis of the ER stress marker protein disulfide isomerase (PDI) at 2, 4, 8 and 24 hours after IsoLG exposure (Figure 6) [49]. Fluorescence immunostaining for PDI revealed increased perinuclear staining of PDI at early time-points in IsoLG treated cells. However, we observed that staining intensity decreased over time and decreased by 24 hours, indicating a reduction of ER stress at the later time-points. To confirm the lack of activation of UPR at later time-points we measured specific RNA and protein indices of UPR activation, namely the chaperone Bip/Grp78, the transcription factors ATF-4 and XBP-1. We detected no significant up-regulation of any of these markers, suggesting that ER stress activation following IsoLG exposure is an early event, which precedes activation of autophagy.

\section{DISCUSSION}

Oxidative stress is a key factor promoting chronic liver damage and fibrosis progression to cirrhosis. Within this general background, the pro-fibrogenic features of IsoLGs are largely unknown although the formation of IsoLGs has been demonstrated by the detection of their 


\section{Longato}

adducts in several animal models of liver disease $[16,28,29,50]$. This paucity of information is largely consequent to the difficulty of precisely assessing the in vivo levels of IsoLG in physiological and pathological conditions, and to the fact that they do not exist in free form due to their extreme rapidity of adduction [16]. The aim of the present study was to achieve a better understanding of the potential IsoLG-related pro-fibrogenic mechanisms and how their formation may contribute to disease progression.

A key issue in the development of the study design was the establishment of the experimental dose range to be employed, and to use concentrations that may be physiologically relevant (i.e. nanomolar concentrations). Along these lines, quantification of circulating levels of IsoLGE ${ }_{2}$-protein adducts in patients with either atherosclerosis or renal disease had been reported in the range of $300 \mathrm{nM}$, i.e. roughly twice those observed in healthy individuals [51]. On the other hand, the concentration of IsoLG-phosphatidylethanolamine (IsoLG-PE) adducts in whole livers of ethanol-fed mice was shown to be approximately $30 \mathrm{nM}$ [28]. In addition, previous experimental evidence obtained in in vitro studies has suggested that IsoLG-PE may have a limited incorporation into the cells leading to the need of employing higher doses in order to elicit biological effects [20]. Based on these considerations, we decided to employ a range of concentrations (picomolar or nanomolar concentrations) which are likely to be reached in conditions of sustained oxidative stress in vivo.

As a general result outcome it is rather clear that, when compared to a classic and well characterized reactive aldehyde such as 4-HNE, IsoLGs present some distinct features at least for what regards their biological effects on HSC, indeed, IsoLG are cytotoxic and pro-apoptotic at much lower concentration than $4-\mathrm{HNE}(5 \mu \mathrm{M}$ versus $>25 \mu \mathrm{M}$ for $4-\mathrm{HNE})$ [7, 39]. These values are in line with what has recently been demonstrated in other cell types [22]. In addition, IsoLG are bioactive at lower concentrations (range: $50 \mathrm{nM}-500 \mathrm{nM}$ ), compared with 1-10 $\mu \mathrm{M}$ for 4-HNE. Similar to 4-HNE, IsoLG had limited or no effect on cell proliferation and DNA synthesis 


\section{Longato}

[7]. In contrast to 4-HNE, IsoLG did not exert a direct pro-fibrogenic effect by inducing collagen synthesis [6, 7]. Despite the lack of a direct effect on collagen production by HSC and thus fibrogenesis, it is worth mentioning that IsoLG-modified proteins can become resistant to proteolysis [18]. Recent work has described how IsoLG exposure results in modifications of collagen $1 \alpha 1$, which render it resistant to matrix metalloproteinase 1 mediated degradation and prone to accumulation thus contributing indirectly to pulmonary fibrosis [22].

Investigation on the intracellular signaling elicited by ROS and reactive aldehydes has proved to be of key importance in order to explain their biological effects [5]. In this regard, IsoLG treatment resulted in the activation of ERK and JNK signaling, and which was sustained at 24 hours post-treatment, but had no effect on either NF-kB or p38 MAPK activation. While 4HNE typically induces JNK and p38 MAPK activation in HSCs $[7,8]$ and in other cell types [39, 52] it does not have significant effects on either Ras/ERK [7] or NF-kB [8]. Considering the lack of IsoLG-mediated NF-kB activation in $\mathrm{hHSC}$ while there is evidence that $15-\mathrm{E}_{2}$-IsoLG strongly activates NF-kB in macrophages, this confirms previous observations suggesting a strong celltype dependency in the response to this mediator [30]. Similarly, we did not detect any significant activation of p38 MAPK, a known target of ER stress activation [20, 47]. Overall, the most profound effect of hHSC exposure to IsoLG is an effect on the synthesis and secretion of pro-inflammatory cytokines/chemokines. Such direct pro-inflammatory and activating effects have also been demonstrated in immune cells such as macrophages [30], and dendritic cells [27]. Whilst HSC are not traditionally considered as the main hepatic immune cell type, they contribute to tissue inflammation by providing chemotactic signals that regulate their interaction with inflammatory cell types, as well as HSC themselves $[53,54]$. Therefore, in the context of oxidative stress during liver injury, IsoLGs formed will promote the pro-inflammatory role of HSC in causing chronic liver injury and inflammation.

An additional important observation of the present study is the dose-dependent increase in intracellular ROS formation resulting from HSC exposure to IsoLG. This could contribute to 


\section{Longato}

the pro-fibrogenic role of HSC biology by multiple mechanisms. These include the activation of redox-dependent stress kinases such as JNK and ERK [55-57], and the stimulation of autophagy $[47,58,59]$. Along these lines, the results of this study provide evidence that IsoLG exposure in HSC activates autophagy. This likely occurs as a compensatory anti-stress response, since inhibition of autophagy by bafilomycin increases the formation of proinflammatory cytokines. Indeed, autophagy can serve as a protective, pro-survival mechanism in cells exposed to oxidant stress, as reported in primary hepatocytes following ischemia/reperfusion [58], and $\mathrm{HSC}$ following $\mathrm{H}_{2} \mathrm{O}_{2}$ treatment [47]. Interestingly, it has been suggested that enhanced autophagy is required to establish an activated phenotype in HSC, by providing energy for the process via lipid droplets mobilization $[45,46]$.

Induction of autophagy can be dependent on ER stress and UPR activation, particularly in the case of the IRE1/Xbp1 branch [47, 52,60]. IsoLG activation appeared to trigger changes in PDI immunofluorescence and distribution between 2-4 hours after exposure, with little or no effect at later time-points, consistent with an earlier activation of ER stress. Indeed, we did not detect any increases in protein or RNA markers of UPR activation at 24 hours, nor ultrastructural evidence of ER stress, such as ER enlargement and swelling and cytoplasmic vacuolization. Collectively, this suggests that autophagy induction by IsoLG is preceded by UPR activation.

The hypothesis that IsoLG exposure may activate autophagy to ameliorate proteotoxicity has recently been proposed in the context of pulmonary fibrosis. In this view, IsoLG cytotoxicity could be the result of excessive accumulation of modified/adducted proteins [22]. Whilst this could also be the case for HSC, it must be noted that recent work has differentiated between the pathogenic role of IsoLG protein- and lipid-, particularly phosphatidylethanolamine, adducts [20, 30, 34]. Our experimental setup, based on the direct treatment with $15-E_{2}-$ IsoLG, which may generate both types of adducts, does not allow us to attribute the observed effects solely to protein adducts and further studies will be necessary for a more precise definition in this sense. 
Longato

In conclusion, the data presented in this work provide the first evidence of the direct effects of chemically synthesized IsoLG on hHSC. In particular, we demonstrate multiple effects of IsoLGs on HSC activation, and particularly increased MAPK activation (ERK/JNK), cytokines and chemokines production, intracellular ROS production, and autophagy induction. Collectively, these data suggest that formation of IsoLGs may be a key pathway linking oxidative injury to disease progression in fibrogenic liver diseases characterised by chronic exposure to oxidative stress products.

\section{ACKNOWLEDGEMENTS}

This work was in part supported by a grant from the NIHR University College London Hospitals Biomedical Research Centre (Award: BRC21/III). LL received additional financial support from the UCL Division of Medicine Athena SWAN Committee. The authors have no other financial disclosures.

\section{AUTHORS DISCLOSURE STATEMENT}

No competing financial interests exist.

\section{LIST OF ABBREVIATIONS}

- $15-E_{2}-$ IsoLG, isolevuglandin $E_{2}$;

- 4-HNE, 4-hydroxy-2-nonenal;

- a-SMA, alpha Smooth Muscle Actin;

- ANOVA, analysis of variance;

- ATF4, Activating transcription factor 4;

- BiP, Immunoglobulin heavy chain binding protein;

- BrdU, 5-Bromo-2-Deoxyuridine;

- carboxy-DCF, (5(6)-Carboxy-2',7'-dichlorofluorescein; 


\section{Longato}

- carboxy- $\mathrm{H}_{2}$ DCFDA, 2',7'-dichlorodihydrofluorescein diacetate;

- CHOP, CCAAT-enhancer-binding protein homologous protein;

- CCL2, Chemokine (C-C motif) ligand 2;

- CM, Complete Medium;

- Col1A1, Collagen type I alpha 1;

- $\mathrm{CQ}$,chloroquine;

- DAPI, 4',6-diamidino-2-phenylindole;

- ELISA, Enzyme-Linked Immunosorbent Assay;

- ER, Endoplasmic Reticulum;

- ERK, extracellular related kinase;

- GAPDH, glyceraldehyde 3-phosphate dehydrogenase;

- HBSS: Hank's balanced salt solution;

- hHSC, Human Hepatic Stellate Cells;

- ICAM-1, intracellular adhesion molecule;

- IL1 $\beta$, Interleukin-1 beta;

- IL6, Interleukin-6;

- IL8, Interleukin-8;

- IsoLG, isolevuglandin;

- IsoK, isoketals;

- JNK, c-jun N-terminal kinase;

- LC3B, light chain myosin 3;

- LDH, lactate dehydrogenase;

- LOX, Lysyl oxidase;

- MTS, (4-sulfophenyl)-2H-tetrazolium, inner salt);

- NF-kB, Nuclear Factor kappa B; 
Longato

- p38MAPK, p38 mitogen-activated protein kinases;

- PARP, poly (ADP-ribose) polymerase;

- PDI, protein disulfide isomerase;

- qRT-PCR, quantitative reverse transcription PCR;

- ROS, Reactive Oxygen Species;

- SEM, Standard Error of the Mean;

- SFM, Serum Free Medium;

- UPR, unfolded protein response;

- TIMP-1, Metallopeptidase inhibitor 1;

- XBP1, X-box binding protein 1.

\section{REFERENCES:}

[1] M. Pinzani, Pathophysiology of Liver Fibrosis, Dig Dis 33(4) (2015) 492-7.

[2] K. Rombouts, V. Carloni, Determination and Characterization of Tetraspanin-Associated Phosphoinositide-4 Kinases in Primary and Neoplastic Liver Cells, Methods Mol Biol 1376 (2016) 203-12.

[3] K. Rombouts, C.R. Gandhi, M. Pinzani, Stellate cells in health and disease, Elsevier : Academic Press, Boston, 2015, pp. xvii, 314 pages.

[4] M. Comporti, B. Arezzini, C. Signorini, C. Sgherri, B. Monaco, C. Gardi, F2-isoprostanes stimulate collagen synthesis in activated hepatic stellate cells: a link with liver fibrosis?, Lab Invest 85(11) (2005) 1381-91.

[5] E. Novo, M. Parola, Redox mechanisms in hepatic chronic wound healing and fibrogenesis, Fibrogenesis Tissue Repair 1(1) (2008) 5. 


\section{Longato}

[6] M. Parola, M. Pinzani, A. Casini, E. Albano, G. Poli, A. Gentilini, P. Gentilini, M.U. Dianzani, Stimulation of lipid peroxidation or 4-hydroxynonenal treatment increases procollagen alpha 1 (I) gene expression in human liver fat-storing cells, Biochem Biophys Res Commun 194(3) (1993) 1044-50.

[7] E. Zamara, E. Novo, F. Marra, A. Gentilini, R.G. Romanelli, A. Caligiuri, G. Robino, E. Tamagno, M. Aragno, O. Danni, R. Autelli, S. Colombatto, M.U. Dianzani, M. Pinzani, M. Parola, 4-Hydroxynonenal as a selective pro-fibrogenic stimulus for activated human hepatic stellate cells, J Hepatol 40(1) (2004) 60-8. [8] M. Parola, G. Robino, F. Marra, M. Pinzani, G. Bellomo, G. Leonarduzzi, P. Chiarugi, S. Camandola, G. Poli, G. Waeg, P. Gentilini, M.U. Dianzani, HNE interacts directly with JNK isoforms in human hepatic stellate cells, J Clin Invest 102(11) (1998) 1942-50.

[9] F. Marra, R. DeFranco, C. Grappone, M. Parola, S. Milani, G. Leonarduzzi, S. Pastacaldi, U.O. Wenzel, M. Pinzani, M.U. Dianzani, G. Laffi, P. Gentilini, Expression of monocyte chemotactic protein-1 precedes monocyte recruitment in a rat model of acute liver injury, and is modulated by vitamin $\mathrm{E}, \mathrm{J}$ Investig Med 47(1) (1999) 66-75.

[10] N. Bernoud-Hubac, S.S. Davies, O. Boutaud, T.J. Montine, L.J. Roberts, 2nd, Formation of highly reactive gamma-ketoaldehydes (neuroketals) as products of the neuroprostane pathway, J Biol Chem 276(33) (2001) 30964-70.

[11] R.G. Salomon, Levuglandins and isolevuglandins: stealthy toxins of oxidative injury, Antioxid Redox Signal 7(1-2) (2005) 185-201.

[12] R.G. Salomon, Distinguishing levuglandins produced through the cyclooxygenase and isoprostane pathways, Chem Phys Lipids 134(1) (2005) 1-20.

[13] R.G. Salomon, G. Subbanagounder, U. Singh, J. O'Neil, H.F. Hoff, Oxidation of low-density lipoproteins produces levuglandin-protein adducts, Chem Res Toxicol 10(7) (1997) 750-9.

[14] S.S. Davies, V. Amarnath, L.J. Roberts, 2nd, Isoketals: highly reactive gamma-ketoaldehydes formed from the H2-isoprostane pathway, Chem Phys Lipids 128(1-2) (2004) 85-99.

[15] O. Boutaud, C.J. Brame, R.G. Salomon, L.J. Roberts, 2nd, J.A. Oates, Characterization of the lysyl adducts formed from prostaglandin H2 via the levuglandin pathway, Biochemistry 38(29) (1999) 9389-96. 


\section{Longato}

[16] C.J. Brame, R.G. Salomon, J.D. Morrow, L.J. Roberts, 2nd, Identification of extremely reactive gamma-ketoaldehydes (isolevuglandins) as products of the isoprostane pathway and characterization of their lysyl protein adducts, J Biol Chem 274(19) (1999) 13139-46.

[17] R.S. Iyer, S. Ghosh, R.G. Salomon, Levuglandin E2 crosslinks proteins, Prostaglandins 37(4) (1989) 471-80.

[18] S.S. Davies, V. Amarnath, K.S. Montine, N. Bernoud-Hubac, O. Boutaud, T.J. Montine, L.J. Roberts, 2nd, Effects of reactive gamma-ketoaldehydes formed by the isoprostane pathway (isoketals) and cyclooxygenase pathway (levuglandins) on proteasome function, FASEB J 16(7) (2002) 715-7.

[19] K.K. Murthi, L.R. Friedman, N.L. Oleinick, R.G. Salomon, Formation of DNA-protein cross-links in mammalian cells by levuglandin E2, Biochemistry 32(15) (1993) 4090-7.

[20] L. Guo, Z. Chen, B.E. Cox, V. Amarnath, R.F. Epand, R.M. Epand, S.S. Davies, Phosphatidylethanolamines modified by gamma-ketoaldehyde (gammaKA) induce endoplasmic reticulum stress and endothelial activation, J Biol Chem 286(20) (2011) 18170-80.

[21] I.G. Stavrovskaya, S.V. Baranov, X. Guo, S.S. Davies, L.J. Roberts, 2nd, B.S. Kristal, Reactive gamma-ketoaldehydes formed via the isoprostane pathway disrupt mitochondrial respiration and calcium homeostasis, Free Radic Biol Med 49(4) (2010) 567-79.

[22] S. Mont, S.S. Davies, L.J. Roberts Second, R.L. Mernaugh, W.H. McDonald, B.H. Segal, W. Zackert, J.A. Kropski, T.S. Blackwell, K.R. Sekhar, J.J. Galligan, P.P. Massion, L.J. Marnett, E.L. Travis, M.L. Freeman, Accumulation of isolevuglandin-modified protein in normal and fibrotic lung, Sci Rep 6 (2016) 24919.

[23] E.J. Carrier, I. Zagol-Ikapitte, V. Amarnath, O. Boutaud, J.A. Oates, Levuglandin forms adducts with histone h4 in a cyclooxygenase-2-dependent manner, altering its interaction with DNA, Biochemistry 53(15) (2014) 2436-41.

[24] S.S. Davies, V. Amarnath, C.J. Brame, O. Boutaud, L.J. Roberts, 2nd, Measurement of chronic oxidative and inflammatory stress by quantification of isoketal/levuglandin gamma-ketoaldehyde protein adducts using liquid chromatography tandem mass spectrometry, Nat Protoc 2(9) (2007) 2079-91. 


\section{Longato}

[25] S.S. Davies, E.J. Brantley, P.A. Voziyan, V. Amarnath, I. Zagol-lkapitte, O. Boutaud, B.G. Hudson, J.A. Oates, L.J. Roberts, 2nd, Pyridoxamine analogues scavenge lipid-derived gamma-ketoaldehydes and protect against H2O2-mediated cytotoxicity, Biochemistry 45(51) (2006) 15756-67.

[26] R.G. Salomon, G. Subbanagounder, J. O'Neil, K. Kaur, M.A. Smith, H.F. Hoff, G. Perry, V.M. Monnier, Levuglandin E2-protein adducts in human plasma and vasculature, Chem Res Toxicol 10(5) (1997) 536-45.

[27] A. Kirabo, V. Fontana, A.P. de Faria, R. Loperena, C.L. Galindo, J. Wu, A.T. Bikineyeva, S. Dikalov, L. Xiao, W. Chen, M.A. Saleh, D.W. Trott, H.A. Itani, A. Vinh, V. Amarnath, K. Amarnath, T.J. Guzik, K.E. Bernstein, X.Z. Shen, Y. Shyr, S.C. Chen, R.L. Mernaugh, C.L. Laffer, F. Elijovich, S.S. Davies, H. Moreno, M.S. Madhur, J. Roberts, 2nd, D.G. Harrison, DC isoketal-modified proteins activate T cells and promote hypertension, J Clin Invest 124(10) (2014) 4642-56.

[28] W. Li, J.M. Laird, L. Lu, S. Roychowdhury, L.E. Nagy, R. Zhou, J.W. Crabb, R.G. Salomon, Isolevuglandins covalently modify phosphatidylethanolamines in vivo: detection and quantitative analysis of hydroxylactam adducts, Free Radic Biol Med 47(11) (2009) 1539-52.

[29] S. Roychowdhury, M.R. McMullen, M.T. Pritchard, W. Li, R.G. Salomon, L.E. Nagy, Formation of gamma-ketoaldehyde-protein adducts during ethanol-induced liver injury in mice, Free Radic Biol Med 47(11) (2009) 1526-38.

[30] L. Guo, Z. Chen, V. Amarnath, P.G. Yancey, B.J. Van Lenten, J.R. Savage, S. Fazio, M.F. Linton, S.S. Davies, Isolevuglandin-type lipid aldehydes induce the inflammatory response of macrophages by modifying phosphatidylethanolamines and activating the receptor for advanced glycation endproducts, Antioxid Redox Signal 22(18) (2015) 1633-45.

[31] V. Amarnath, K. Amarnath, T. Masterson, S. Davies, L.J. Roberts, A simplified synthesis of the diastereomers of Levuglandin E-2, Synthetic Commun 35(3) (2005) 397-408.

[32] I. Mederacke, D.H. Dapito, S. Affo, H. Uchinami, R.F. Schwabe, High-yield and high-purity isolation of hepatic stellate cells from normal and fibrotic mouse livers, Nat Protoc 10(2) (2015) 305-15.

[33] G. Robino, M. Parola, F. Marra, A. Caligiuri, R.M. De Franco, E. Zamara, G. Bellomo, P. Gentilini, M. Pinzani, M.U. Dianzani, Interaction between 4-hydroxy-2,3-alkenals and the platelet-derived growth 


\section{Longato}

factor-beta receptor. Reduced tyrosine phosphorylation and downstream signaling in hepatic stellate cells, J Biol Chem 275(51) (2000) 40561-7.

[34] C.B. Sullivan, E. Matafonova, L.J. Roberts, 2nd, V. Amarnath, S.S. Davies, Isoketals form cytotoxic phosphatidylethanolamine adducts in cells, J Lipid Res 51(5) (2010) 999-1009.

[35] G. Repetto, A. del Peso, J.L. Zurita, Neutral red uptake assay for the estimation of cell viability/cytotoxicity, Nat Protoc 3(7) (2008) 1125-31.

[36] T.D. Schmittgen, K.J. Livak, Analyzing real-time PCR data by the comparative C(T) method, Nat Protoc 3(6) (2008) 1101-8.

[37] A.P. Holt, E.L. Haughton, P.F. Lalor, A. Filer, C.D. Buckley, D.H. Adams, Liver myofibroblasts regulate infiltration and positioning of lymphocytes in human liver, Gastroenterology 136(2) (2009) 70514.

[38] K. Uchida, M. Shiraishi, Y. Naito, Y. Torii, Y. Nakamura, T. Osawa, Activation of stress signaling pathways by the end product of lipid peroxidation. 4-hydroxy-2-nonenal is a potential inducer of intracellular peroxide production, J Biol Chem 274(4) (1999) 2234-42.

[39] P.V. Usatyuk, N.L. Parinandi, V. Natarajan, Redox regulation of 4-hydroxy-2-nonenal-mediated endothelial barrier dysfunction by focal adhesion, adherens, and tight junction proteins, J Biol Chem $281(46)(2006)$ 35554-66.

[40] E. Vladykovskaya, S.D. Sithu, P. Haberzettl, N.S. Wickramasinghe, M.L. Merchant, B.G. Hill, J. McCracken, A. Agarwal, S. Dougherty, S.A. Gordon, D.A. Schuschke, O.A. Barski, T. O'Toole, S.E. D'Souza, A. Bhatnagar, S. Srivastava, Lipid peroxidation product 4-hydroxy-trans-2-nonenal causes endothelial activation by inducing endoplasmic reticulum stress, J Biol Chem 287(14) (2012) 11398-409.

[41] R.G. Salomon, W. Bi, Isolevuglandin adducts in disease, Antioxid Redox Signal 22(18) (2015) 170318.

[42] J.J. Driscoll, R.D. Chowdhury, Molecular crosstalk between the proteasome, aggresomes and autophagy: translational potential and clinical implications, Cancer Lett 325(2) (2012) 147-54.

[43] V.I. Korolchuk, F.M. Menzies, D.C. Rubinsztein, Mechanisms of cross-talk between the ubiquitinproteasome and autophagy-lysosome systems, FEBS Lett 584(7) (2010) 1393-8. 


\section{Longato}

[44] Y. Kabeya, N. Mizushima, T. Ueno, A. Yamamoto, T. Kirisako, T. Noda, E. Kominami, Y. Ohsumi, T. Yoshimori, LC3, a mammalian homologue of yeast Apg8p, is localized in autophagosome membranes after processing, EMBO J 19(21) (2000) 5720-8.

[45] L.F. Thoen, E.L. Guimaraes, L. Dolle, I. Mannaerts, M. Najimi, E. Sokal, L.A. van Grunsven, A role for autophagy during hepatic stellate cell activation, J Hepatol 55(6) (2011) 1353-60.

[46] V. Hernandez-Gea, Z. Ghiassi-Nejad, R. Rozenfeld, R. Gordon, M.I. Fiel, Z. Yue, M.J. Czaja, S.L. Friedman, Autophagy releases lipid that promotes fibrogenesis by activated hepatic stellate cells in mice and in human tissues, Gastroenterology 142(4) (2012) 938-46.

[47] V. Hernandez-Gea, M. Hilscher, R. Rozenfeld, M.P. Lim, N. Nieto, S. Werner, L.A. Devi, S.L. Friedman, Endoplasmic reticulum stress induces fibrogenic activity in hepatic stellate cells through autophagy, J Hepatol 59(1) (2013) 98-104.

[48] H.O. Rashid, R.K. Yadav, H.R. Kim, H.J. Chae, ER stress: Autophagy induction, inhibition and selection, Autophagy 11(11) (2015) 1956-1977.

[49] M. Salazar, S. Hernandez-Tiedra, S. Torres, M. Lorente, M. Guzman, G. Velasco, Detecting autophagy in response to ER stress signals in cancer, Methods Enzymol 489 (2011) 297-317.

[50] X. Li, Y. Wang, H. Wang, C. Huang, Y. Huang, J. Li, Endoplasmic reticulum stress is the crossroads of autophagy, inflammation, and apoptosis signaling pathways and participates in liver fibrosis, Inflamm Res 64(1) (2015) 1-7.

[51] R.G. Salomon, E. Batyreva, K. Kaur, D.L. Sprecher, M.J. Schreiber, J.W. Crabb, M.S. Penn, A.M. DiCorletoe, S.L. Hazen, E.A. Podrez, Isolevuglandin-protein adducts in humans: products of free radicalinduced lipid oxidation through the isoprostane pathway, Biochim Biophys Acta 1485(2-3) (2000) 225-35.

[52] P. Haberzettl, B.G. Hill, Oxidized lipids activate autophagy in a JNK-dependent manner by stimulating the endoplasmic reticulum stress response, Redox Biol 1 (2013) 56-64.

[53] F. Marra, F. Tacke, Roles for chemokines in liver disease, Gastroenterology 147(3) (2014) 577-594 e1.

[54] E. Seki, R.F. Schwabe, Hepatic inflammation and fibrosis: functional links and key pathways, Hepatology 61(3) (2015) 1066-79. 


\section{Longato}

[55] E. Novo, C. Busletta, L.V. Bonzo, D. Povero, C. Paternostro, K. Mareschi, I. Ferrero, E. David, C. Bertolani, A. Caligiuri, S. Cannito, E. Tamagno, A. Compagnone, S. Colombatto, F. Marra, F. Fagioli, M. Pinzani, M. Parola, Intracellular reactive oxygen species are required for directional migration of resident and bone marrow-derived hepatic pro-fibrogenic cells, J Hepatol 54(5) (2011) 964-74.

[56] E. Novo, D. Povero, C. Busletta, C. Paternostro, L.V. di Bonzo, S. Cannito, A. Compagnone, A. Bandino, F. Marra, S. Colombatto, E. David, M. Pinzani, M. Parola, The biphasic nature of hypoxiainduced directional migration of activated human hepatic stellate cells, J Pathol 226(4) (2012) 588-97.

[57] E. Seki, D.A. Brenner, M. Karin, A liver full of JNK: signaling in regulation of cell function and disease pathogenesis, and clinical approaches, Gastroenterology 143(2) (2012) 307-20.

[58] R.H. Bhogal, C.J. Weston, S.M. Curbishley, D.H. Adams, S.C. Afford, Autophagy: a cyto-protective mechanism which prevents primary human hepatocyte apoptosis during oxidative stress, Autophagy 8(4) (2012) 545-58.

[59] J. Navarro-Yepes, M. Burns, A. Anandhan, O. Khalimonchuk, L.M. del Razo, B. Quintanilla-Vega, A. Pappa, M.I. Panayiotidis, R. Franco, Oxidative stress, redox signaling, and autophagy: cell death versus survival, Antioxid Redox Signal 21(1) (2014) 66-85.

[60] D. Senft, Z.A. Ronai, UPR, autophagy, and mitochondria crosstalk underlies the ER stress response, Trends Biochem Sci 40(3) (2015) 141-8.

Table 1: List of antibodies used in the study

\begin{tabular}{|l|l|l|}
\hline Antibody & Cat No & Source \\
\hline Smooth alpha actin & A2547 & Sigma \\
\hline Cleaved PARP (D214) & $9544 S$ & Cell Signaling technology \\
\hline PARP & ab6079 & Abcam \\
\hline PhosphoSAPK/JNK(Thr183/tyr185) & $4668 S$ & Cell Signaling technology \\
\hline 81E11) & & Cell Signaling technology \\
\hline SAPK/JNK(56G8) & $9258 S$ & \\
\hline
\end{tabular}


Longato

\begin{tabular}{|c|c|c|}
\hline ATF4 & 11815 & Cell Signaling technology \\
\hline Bip/GRP78 & 3183 & Cell Signaling technology \\
\hline ERK $1 / 2$ & 4695 & Cell Signaling technology \\
\hline pERK $1 / 2$ & 4370 & Cell Signaling technology \\
\hline P38 MAPK & 9212 & Cell Signaling technology \\
\hline pP38 MAPK(Thr180/Tyr182) & 4511 & Cell Signaling technology \\
\hline $\mathrm{CHOP}$ & 2895 & Cell Signaling technology \\
\hline Tubulin & 2144 & Cell Signaling technology \\
\hline Donkey anti-goat lgG-HRP & sc-2020 & Santa Cruz Biotechnology \\
\hline Goat anti-mouse IgG-HRP & sc-2055 & Santa Cruz Biotechnology \\
\hline Goat anti-rabbit lgG-HRP & sc-2054 & Santa Cruz Biotechnology \\
\hline anti-mouse LC3B antibody & 3868 & Cell Signaling \\
\hline PDI (Alexa Fluor $\circledast 488$ Conjugate) & 5051 & Cell Signaling \\
\hline
\end{tabular}

Table 2: List of $\operatorname{Taqman}^{\circledR}$ assays used in the study

\begin{tabular}{|l|l|l|}
\hline Gene & Catalogue number & Source \\
\hline GAPDH & Hs02758991_g1 & Life Technologies LTD \\
\hline Collagen 1a1 & Hs00164004_m1 & Life Technologies LTD \\
\hline TIMP-1 & Hs00171558_m1 & Life Technologies LTD \\
\hline LOX & Hs00942480_m1 & Life Technologies LTD \\
\hline ICAM-1 & Hs00164932_m1 & Life Technologies LTD \\
\hline Interleukin 6 (IL-6) & Hs00985639_m1 & Life Technologies LTD \\
\hline MCP-1/CCL2 & Hs00234140_m1 & Life Technologies LTD \\
\hline
\end{tabular}


Longato

\begin{tabular}{|l|l|l|}
\hline Interleukin 8 (IL-8) & Hs00174103_m1 & Life Technologies LTD \\
\hline Interleukin 1 $\beta(\mathrm{IL}-1 \beta)$ & Hs01555410_m1 & Life Technologies LTD \\
\hline ATF-4 & Hs00909569_g1 & Life Technologies LTD \\
\hline XBP-1 & Hs00231936_m1 & Life Technologies LTD \\
\hline
\end{tabular}

\section{FIGURE LEGENDS:}

Figure 1: Human hepatic stellate cells (hHSC) were treated with the indicated doses of $15-\mathrm{E}_{2}$-IsoLG (levuglandin) for 24-48 hrs. Cytotoxicity was assessed by lactate dehydrogenase leakage in culture supernatants (A-B), formazan reduction (MTS assay, C-D), and neutral red dye incorporation (E-F). Cell proliferation was assessed by BrdU ELISA (G-H). Graphs represent averages \pm SEM of four replicates. Data were analyzed via one-way ANOVA with Dunnett's multiple comparisons test. $\mathrm{P}$ values to indicate significance are referred to serum free medium (SFM) treated cells $\left({ }^{*}=\mathrm{P}<0.05,{ }^{* * * *}=\mathrm{P}<0.001\right)$. As the $\mathrm{LDH}$ assay used was not compatible with serum containing medium, the CM samples are not included in these graphs.

Figure 2: High dose IsoLGs cytotoxicity is mediated by activation of apoptosis. hHSC

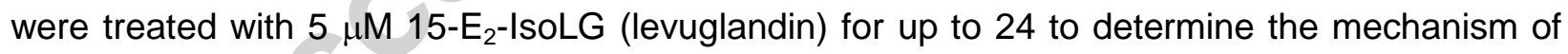
cytotoxicity. Protein lysates collected at different time-points were analyzed for markers of apoptotic cell death via Western Blot (cleaved PARP, GADD153/CHOP, phosphorylated JNK), and normalized to tubulin as loading control.

Figure 3: Exposure to IsoLGs in hHSC promotes the activation of different signaling pathways and $\alpha$-SMA, but has limited effect on markers of fibrogenic activation. hHSC were treated with $0.5 \mu \mathrm{M}-0.5 \mathrm{nM} 15-\mathrm{E}_{2}$-IsoLG (levuglandin) for up to 24 . mRNA markers of fibrogenesis including collagen 1 alpha 1, tissue inhibitor of metalloproteinase 1, and lysyl 


\section{Longato}

oxidase (LOX), were measured via qRT-PCR, and normalized to GAPDH as internal control. The activation of different signaling pathways and smooth muscle actin (aSMA) levels were measured via Western Blot. Protein lysates were analyzed for phosphorylated/total levels of ERK, JNK, P38MAPK, and total levels, normalized to tubulin as loading control.

Figure 4: Exposure to IsoLGs in hHSC promotes the activation of pro-inflammatory signaling pathways and production of ROS. hHSC were treated with non-cytotoxic doses of 15$E_{2}$-IsoLG for up to 24 and qRT-PCR was used to measure gene expression for selected adhesion molecules,cytokines and chemokines, normalized to GAPDH as internal control (A-E). Levels of secreted bioactive IL1 $\beta$ were measured in supernatants of cells treated for 48 hours, using HEK-Blue Cells and QuantiBlue assay (F). In a subset of experiments, mRNA expression was quantified in IsoLG treated cells that were pre-treated for one hour with inhibitors of selected signaling pathways, namely JNK (SP600125, $20 \mu \mathrm{M})$, p38 MAPK (SB203580, $10 \mu \mathrm{M})$, and NF-KB (Pyrrolidinedithiocarbamate, PDTC, $50 \mu \mathrm{M})(\mathrm{G})$. Data are represented either as fold change with respect to SFM treated cells (calibrator), or as relative expression, and analysed via either one way ANOVA, or two-way ANOVA with Dunnett's or Bonferroni's multiple comparisons

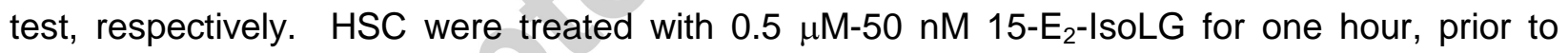
incubation with the carboxy- $\mathrm{H}_{2}$ DCFDA probe to detect intracellular reactive oxygen species (ROS) formation (H). Cells positive for ROS production display green fluorescence, whereas total cell distribution is indicated by Hoechst blue staining as assessed by fluorescence microscope.

Figure 5: IsoLGs exposure in HSC results in activation of autophagy. hHSC were treated with selected doses of $15-E_{2}$-IsoLG for up to 24-48 hours. Representative TEM images

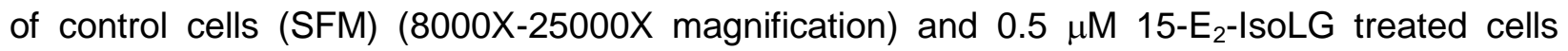
(25000X-50000X magnification) for 24 (left panels) and 48 hours (right panels). Ultrastructural analyses reveal markedly increased presence of multilamellar autophagic vescicles in IsoLGs 
Longato

treated cells, indicative of autophagy (black arrows). Ongoing autophagy was further confirmed by immunofluorescence staining for LC3B puncta (middle panels), in both HSC treated with IsoLGs, and with $15 \mu \mathrm{M}$ chloroquine (CQ) as positive control. Lastly, autophagy was confirmed with Western blot analysis for LC3BII conversion. In a subset of experiments, $15-\mathrm{E}_{2}$-IsoLG treated cells were treated with bafilomycin for 24 hours to measure the levels of LC3B and of IL$1 \beta$ by Western Blot and qRT-PCR, respectively.

Figure 6: IsoLGs exposure in HSC results in early activation of ER stress and unfolded protein response. hHSC were treated with selected doses of $15-\mathrm{E}_{2}$-IsoLG for 2,4,8, and 24 hours. Levels and distribution of protein disulfide isomerase (PDI) were assessed via direct immunofluorescence. Protein and mRNA levels of selected markers of the UPR were measured in 15- $E_{2}$-IsoLG treated cells for 24 hours. 
Longato

24 hours
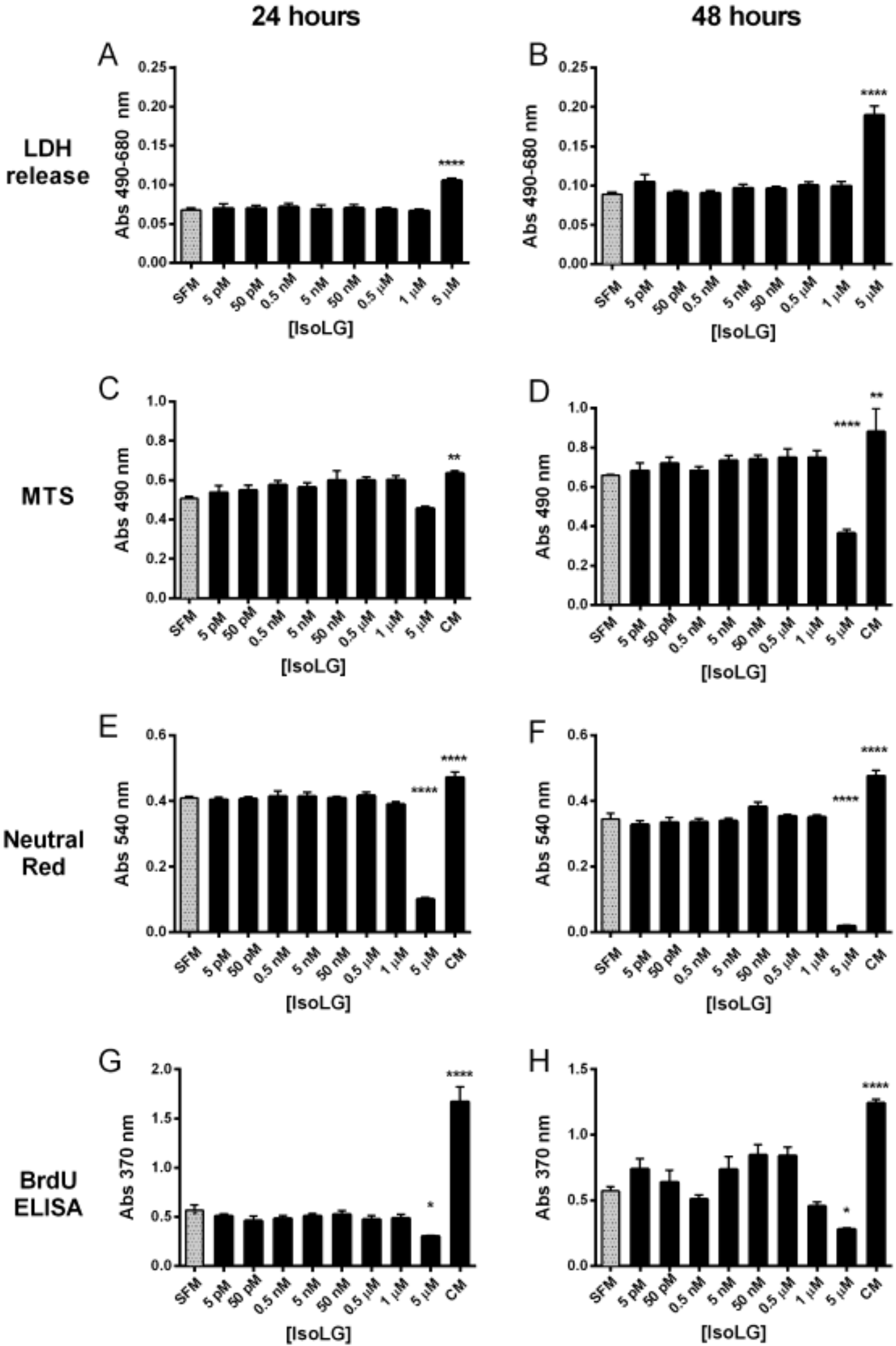
Longato

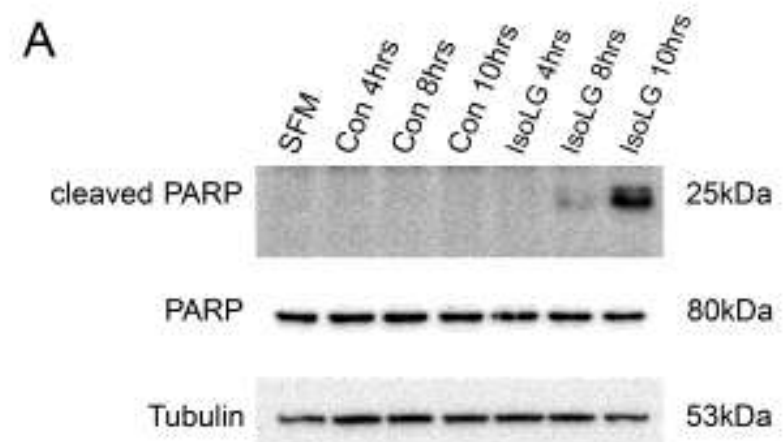

B

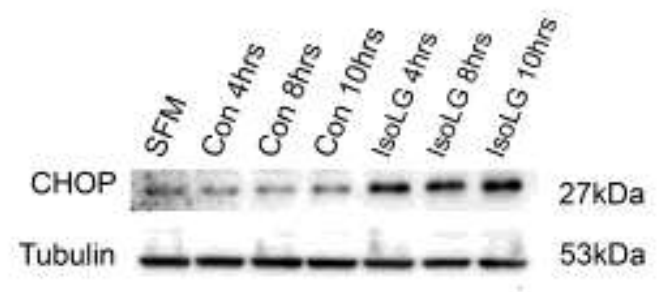

C

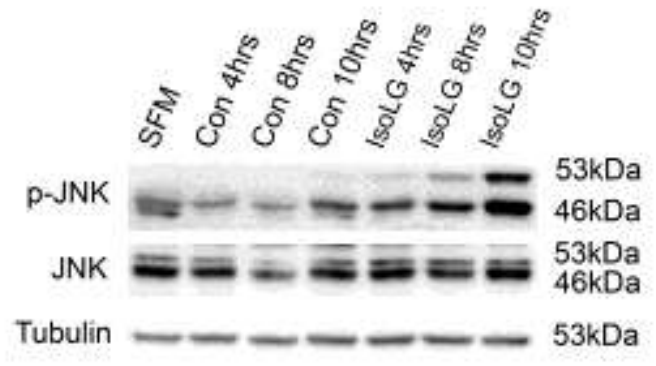


Longato

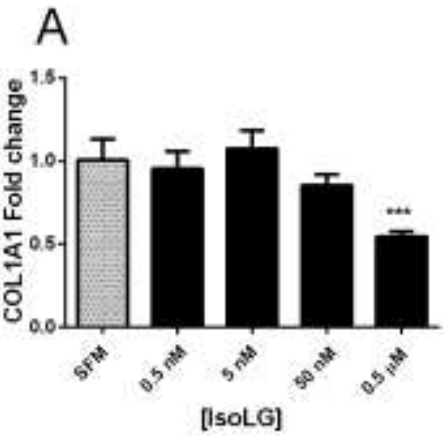

D

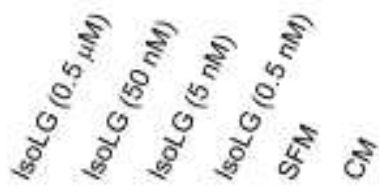

p-Erk

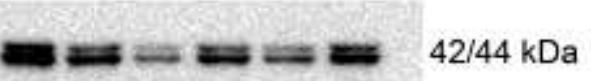

Erk $-\mathrm{z}=\mathrm{z}=42 / 44 \mathrm{kDa}$

ubulin

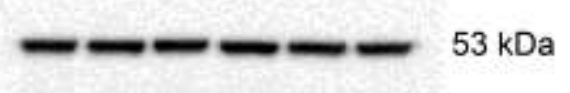

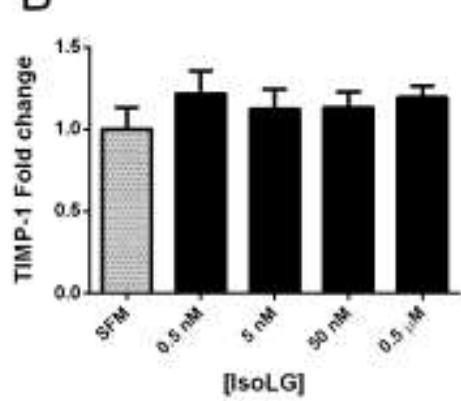

C

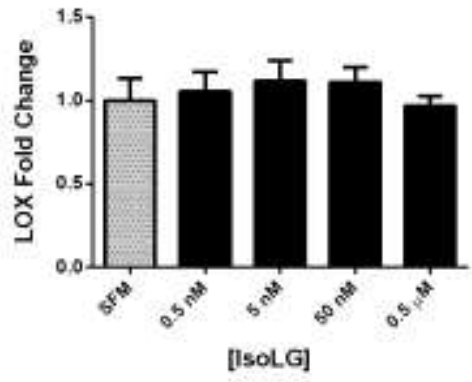

E

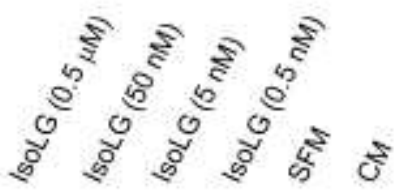

P-JNK

JNK

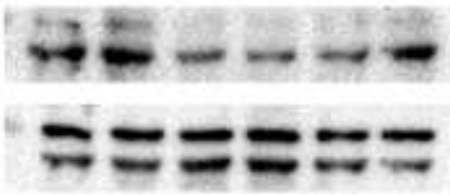

$53 / 46 \mathrm{kDa}$

Tubulin

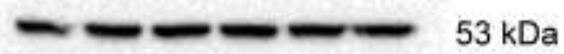

$53 / 46 \mathrm{kDa}$

G

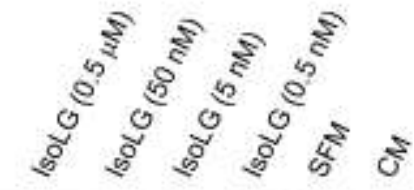

$\alpha$-SMA 42/44 kDa

P-38

$53 \mathrm{kDa}$ 


\section{Longato}

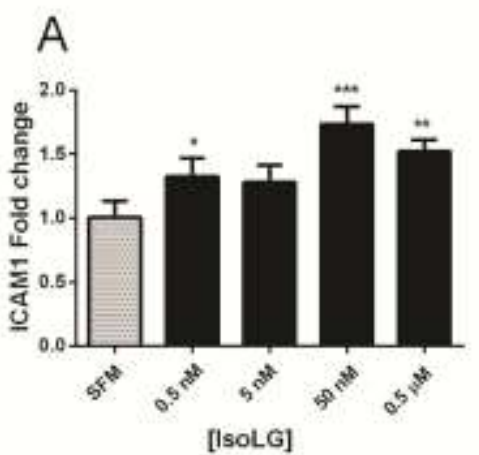

D

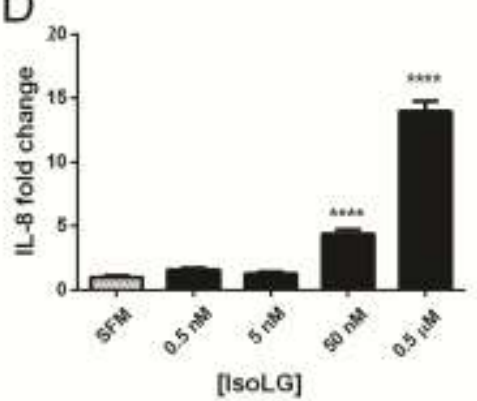

F

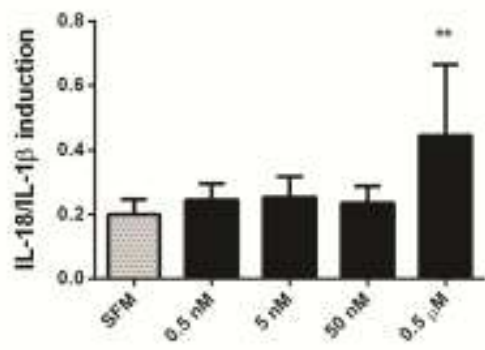

C
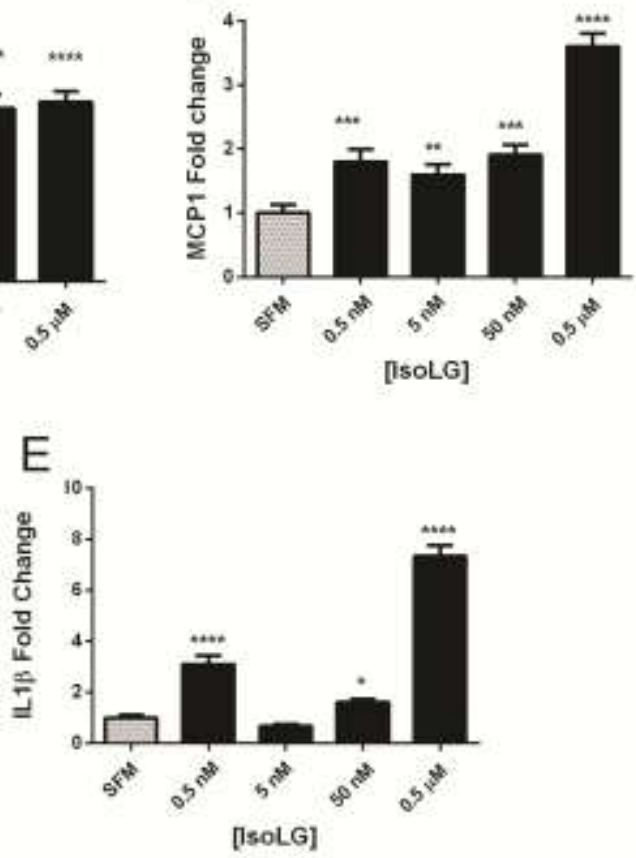

G

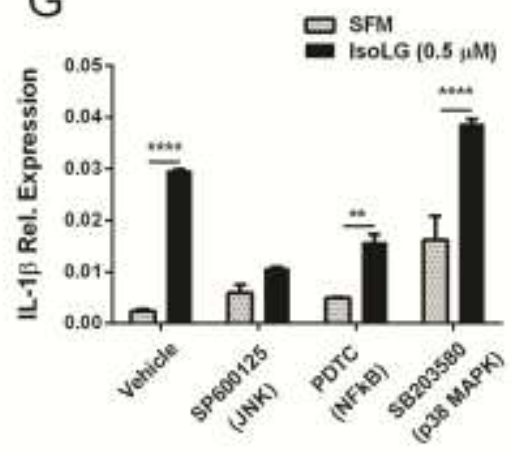

$\mathrm{H}$

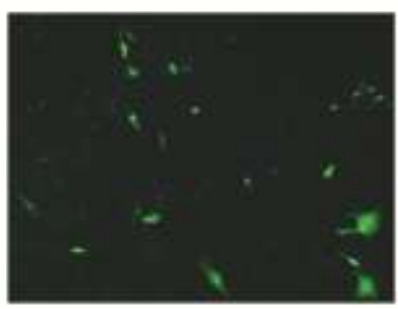

IsoLG (50 nM)

IsoLG $(0.5 \mu \mathrm{M})$

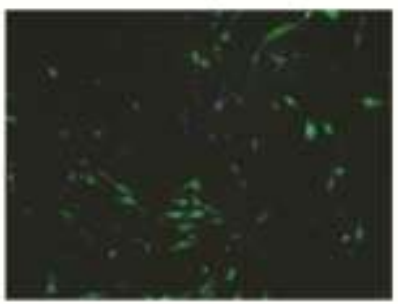

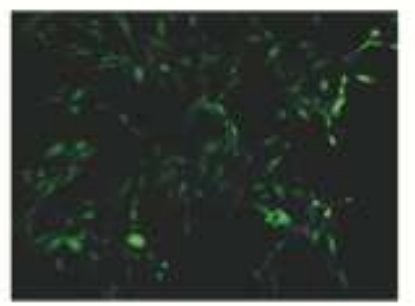


Longato

A

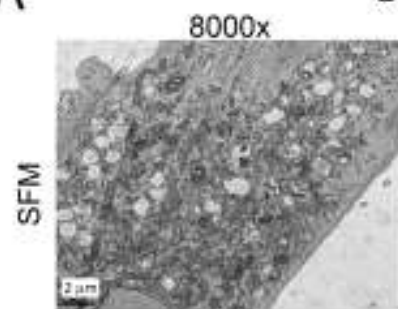

$25000 x$

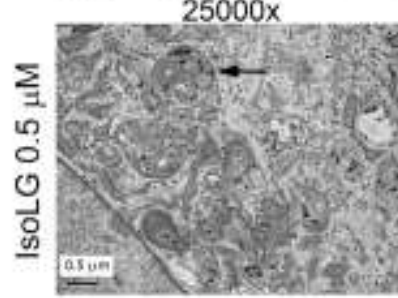

B
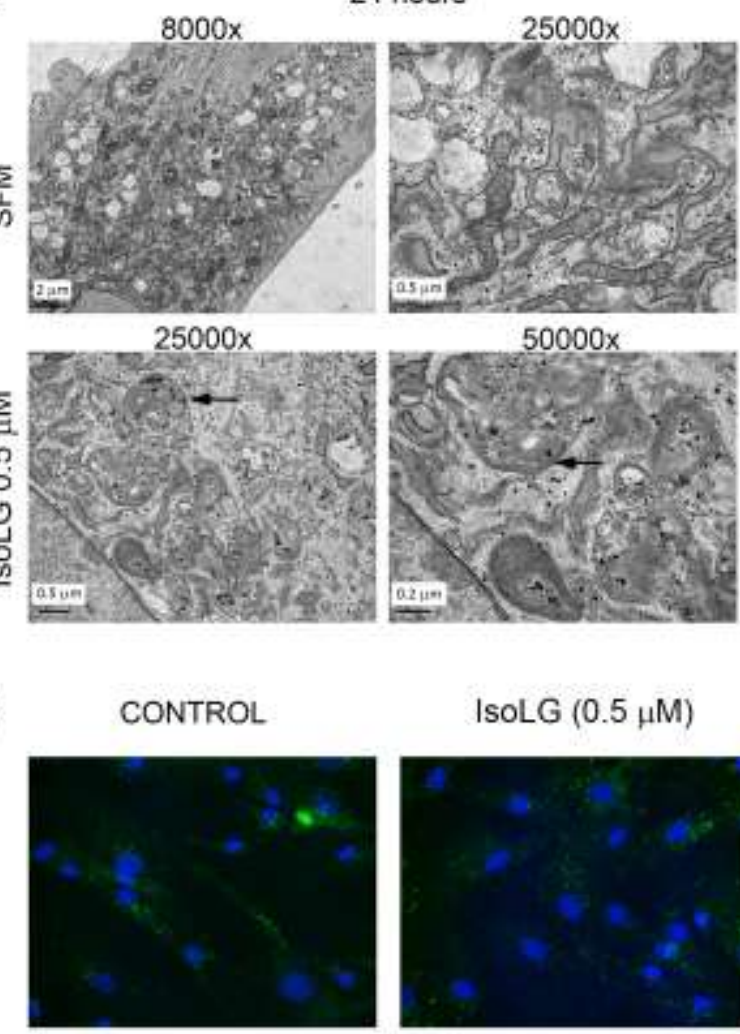

$50000 x$

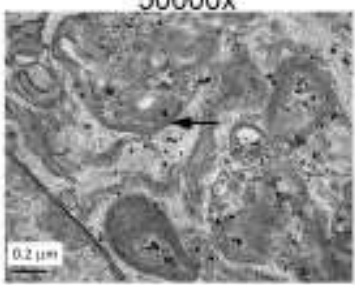

IsoLG $(0.5 \mu \mathrm{M})$

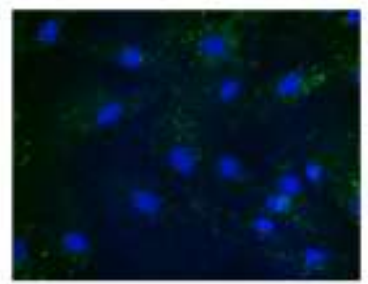

C

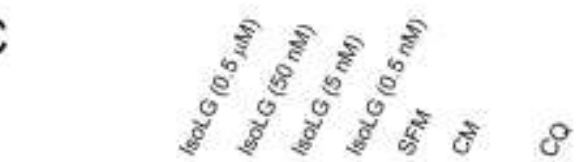

LC3B-I

LC3B-II
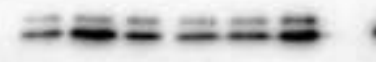

8

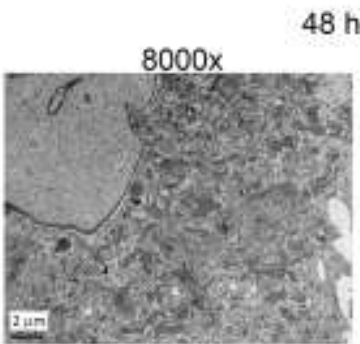

48 hours
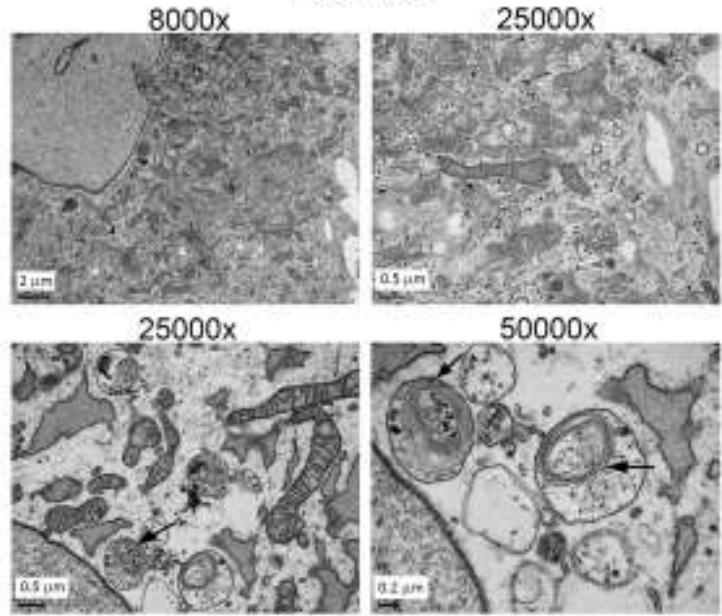

IsoLG (50 nM)

\section{$C Q$ positive control}
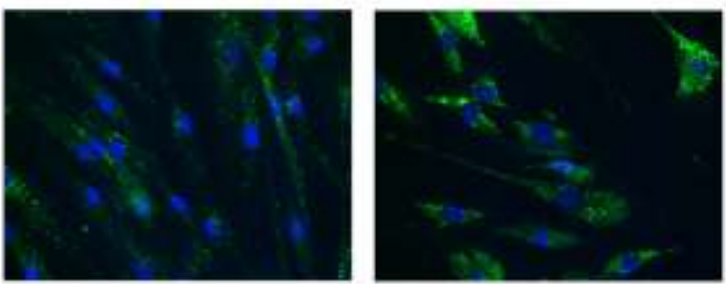

D

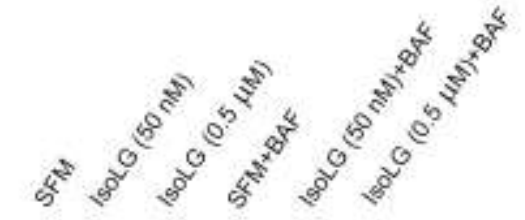

Tubulin

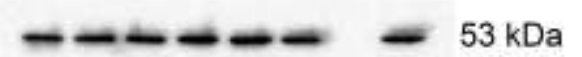

LC3B-I

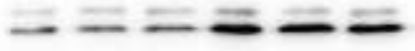

14/16 kDa

E

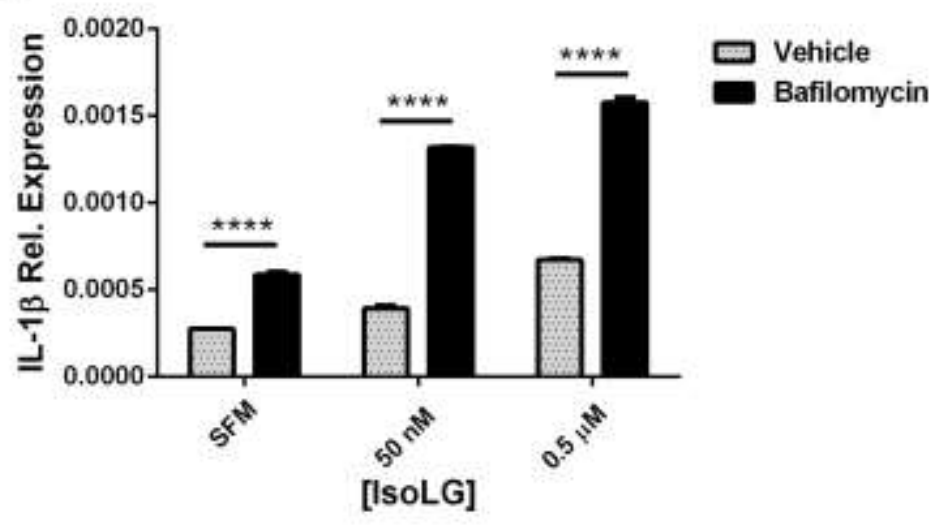


Longato

A
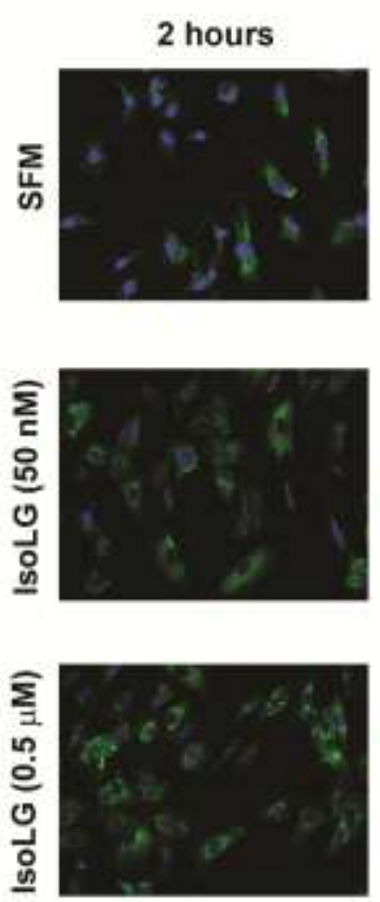

4 hours
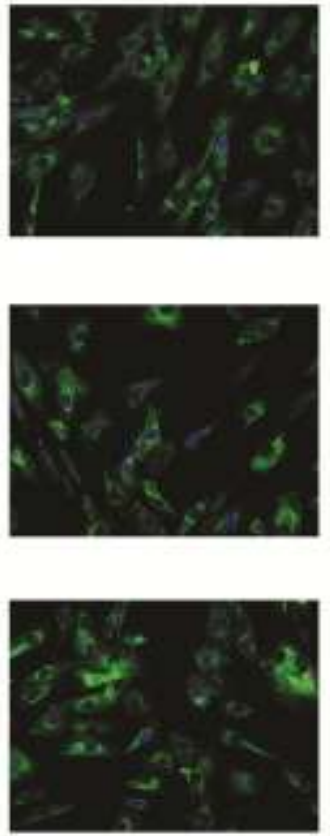

8 hours
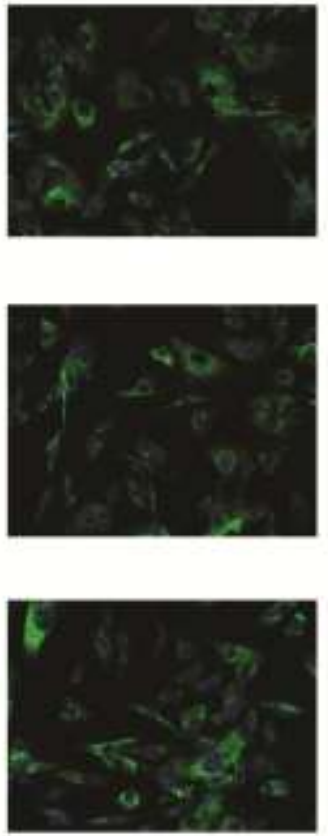

24 hours
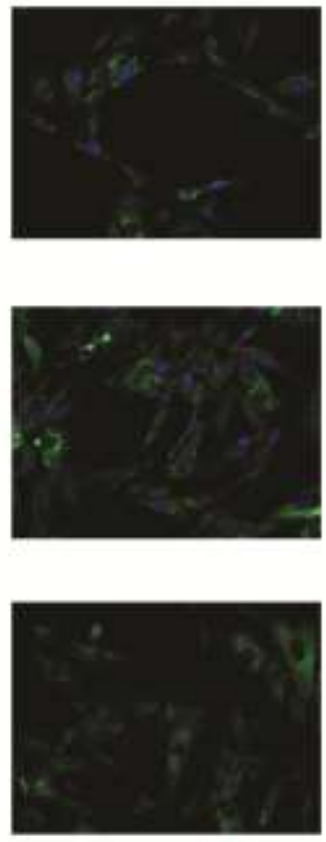

C
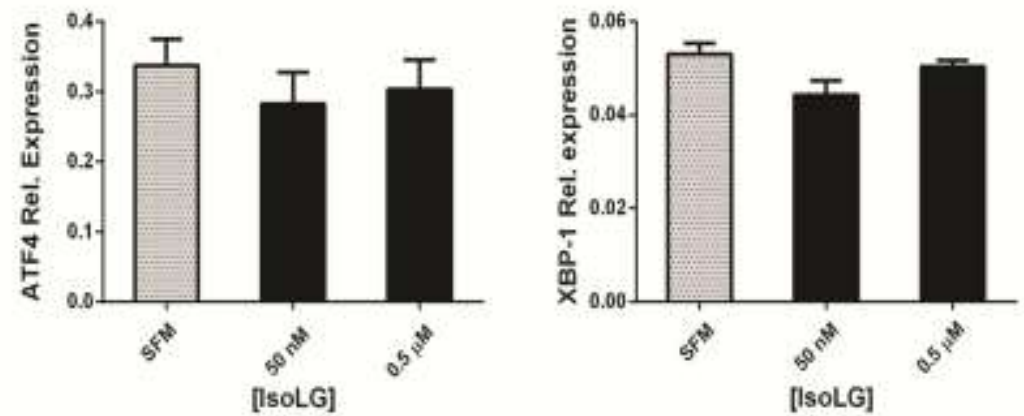

Tubulin

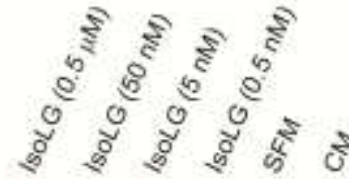

ATF4

Tubulin

BIP

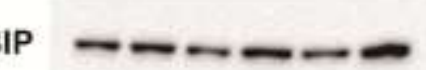

B

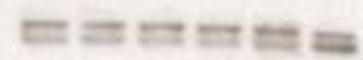

$\longrightarrow-\omega$ 


\section{Longato}

Highlights

- Isolevuglandins (IsoLG) are described as novel activators of hepatic stellate cells

- IsoLG are biologically active at concentrations as low as 500 pM

- IsoLG promote activation of stress signaling pathways and redox changes

- IsoLG induce a pro-inflammatory effect, in part mediated by autophagy activation

- Autophagy may reduce toxicity from IsoLG adduction to cellular proteins 